\title{
SEMISTABLE BUNDLES ON CURVES AND IRREDUCTIBLE REPRESENTATIONS OF THE FUNDAMENTAL GROUP
}

\author{
HÉLÈNE ESNAULT AND ECKART VIEHWEG
}

\section{INTRODUCTION}

In this note, we make an attempt to understand the meaning of Bolibruch's theorem for curves of higher genus.

Theorem 0.1 (Bolibruch [1]). Let

$$
\rho: \pi_{1}\left(\mathbb{P}_{\mathbb{C}}^{1}-\Sigma\right) \longrightarrow G L(N, \mathbb{C})
$$

be an irreducible representation of the fundamental group of the complement of finitely many points $\Sigma \neq \emptyset$. Then there is a logarithmic connection

$$
\nabla: \mathcal{O}^{\oplus N} \longrightarrow \Omega_{X}^{1}(\log \Sigma) \otimes \mathcal{O}^{\oplus N}
$$

such that the local system $\operatorname{ker}\left(\left.\nabla\right|_{X-\Sigma}\right)$ on $\mathbb{P}_{\mathbb{C}}^{1}-\Sigma$ is defined by $\rho$.

Bolibruch's proof is very analytic, but Gabber ([2] gave a more algebraic approach, which we recall in section 1 (see also [4]). Using his construction, we interpret Bolibruch's theorem in the following way.

Theorem 0.2. Let $X$ be a curve over an algebraically closed field $k$ of characteristic 0 , and let $\emptyset \neq \Sigma \subset X(k)$ consist of finitely many points. Let

$$
\nabla: E \longrightarrow \Omega_{X}^{1}(\log \Sigma) \otimes E
$$

be a logarithmic connection on a vectorbundle $E$ of rank $N$ such that for all subsheaves $\{0\} \neq F \subset E$ with $\operatorname{rank}(F)<N$,

$$
\nabla F \not \subset \Omega_{X}^{1}(\log \Sigma) \otimes F \text {. }
$$

Then for any $p \in \Sigma$, there is a semistable vectorbundle $E^{\prime}$ of degree 0 and a logarithmic connection

$$
\nabla^{\prime}: E^{\prime} \longrightarrow \Omega_{X}^{1}(\log \Sigma) \otimes E^{\prime}
$$

with $\left.\left(E^{\prime}, \nabla^{\prime}\right)\right|_{X-\{p\}}=\left.(E, \nabla)\right|_{X-\{p\}}$.

Any semistable bundle $E^{\prime}$ of rank $N$ and degree 0 , has a canonical filtration (see (3.3)), the graded bundles $g r_{i} E^{\prime}$ of which are direct sums of stable ones. Due to the Narasimhan-Seshadri correspondence [5] over $\mathbb{C}$, there is a unitary connection $d_{i}$ on $g r E_{i}^{\prime}$ which is uniquely defined.

The curious point is that, over $k=\mathbb{C}$, we associate to an irreducible representation of the fundamental group

$$
\pi_{1}(X-\Sigma) \longrightarrow G L(N, \mathbb{C})
$$

This work has been partly supported by the DFG Forschergruppe "Arithmetik und Geometrie". 
of the open curve $X-\Sigma$, unitary representations of the fundamental group of the compact curve

$$
\pi_{1}(X) \longrightarrow U\left(N_{i}, \mathbb{C}\right), \text { where } \sum_{i} N_{i}=N
$$

via theorem 0.2 and the Narasimhan-Seshadri correspondence.

Conversely it is easy to associated such unitary representations of $\pi_{1}(X)$ an irreducible representation $\pi_{1}(X-\Sigma) \rightarrow G L(N, \mathbb{C})$ :

Proposition 0.3. Let $X$ be a curve over $\mathbb{C}$ let $E$ be a semistable bundle on $X$ of degree 0 with graded bundles $\mathrm{gr}_{i}(E)$ for the canonical filtration.

1) There is a connection $\nabla: E \rightarrow \Omega_{X}^{1} \otimes E$ respecting the canonical filtration on $E$, such that $\operatorname{gr}_{i}(\nabla)=d_{i}$.

2) There is a constant $\sigma \leq 3$ depending only on $E$ such that for any reduced divisor $\Sigma$ with $\operatorname{deg}(\Sigma) \geq \sigma$, there is a connection

$$
\nabla: E \longrightarrow \Omega_{X}^{1}(\log \Sigma) \otimes E
$$

such that for all subsheaves $\{0\} \neq F \subset E$ with $\operatorname{rank}(F)<N$,

$$
\nabla F \not \subset \Omega_{X}^{1}(\log \Sigma) \otimes F \text {. }
$$

This way of going back and forth between representations of the projective and the open curve is very lose. On both sides one has parameters. It is not clear whether one should think of this really as a correspondence. It is also not clear how to interpret this in terms of compactification of the moduli space of stable bundles of degree 0 .

\section{GabBer's COnstruction}

We explain Gabber's construction, transposing it to the algebraic context of theorem 0.2 . Hence we consider a projective curve $X$ over $k$, a divisor $\Sigma>0$ and a logarithmic connection

$$
\nabla: E \longrightarrow \Omega_{X}^{1}(\log \Sigma) \otimes E
$$

on a vectorbundle $E$. We fix a point $p \in \Sigma$ and denote by

$$
\Gamma=\operatorname{res}_{p}(\nabla): E \otimes k(p) \longrightarrow E \otimes k(p)
$$

the residue of $\nabla$.

For $0 \neq w \in E \otimes k(p)$ define $E_{w}^{\prime}$ to be the inverse image of $k_{w}$ under the restriction map $E \rightarrow E \otimes k(p)$, and $E_{w}=E_{w}^{\prime}(p)$. Then $E \subset E_{w} \subset E(p)$ and $\operatorname{deg} E_{w}=\operatorname{deg}(E)+1$.

The connection $\nabla$ extends to $\nabla_{w}$ on $E_{w}$ if and only if $w$ is an eigenvector of $\Gamma$. More precisely, let $\left(w, e_{2}, \ldots, e_{N}\right)$ be a basis of $E \otimes k(p)$ in which $\Gamma=\left(\gamma_{i j}\right)$ is triangular, that is $\gamma_{i j}=0 i>j$. Then in the basis $\left(\frac{w}{t}, e_{2}, \ldots, e_{N}\right)$ of $E_{w} \otimes k(p)$ the residue $\operatorname{res}_{p}\left(\nabla_{w}\right)=\Gamma_{w}=\left(\gamma_{i j}^{\prime}\right)$ fulfills:

$$
\begin{aligned}
& \gamma_{i j}^{\prime}=\gamma_{i j} \text { for } i \geq 2, j \geq 2 \\
& \gamma_{11}^{\prime}=\gamma_{11}-1 \\
& \gamma_{1 i}^{\prime}=0 i \geq 2 .
\end{aligned}
$$


Thus the roots of the characteristic polynomial of $\Gamma_{w}$, are $\gamma_{11}-1, \gamma_{22}, \ldots, \gamma_{N N}$.

Definition 1.1. We say that $\left(E^{\prime}, \nabla^{\prime}\right)$ is obtained from $(E, \nabla)$ by an elementary $G$-transformation at $p$ if there is an eigenvector $0 \neq w \in E \otimes k(p)$ of $\Gamma$ such that $\left(E^{\prime}, \nabla^{\prime}\right)=\left(E_{w}, \nabla_{w}\right)$.

Theorem 1.2 (Gabber). Let $\nabla: E \rightarrow \Omega_{X}^{1}(\log \Sigma) \otimes E$ be any connection, and $M \in \mathbb{N}$. Then there is a connection

$$
\nabla^{\prime}: E^{\prime} \longrightarrow \Omega_{X}^{1}(\log \Sigma) \otimes E^{\prime}
$$

with $\left.\left(E^{\prime}, \nabla^{\prime}\right)\right|_{X-\{p\}}=\left.(E, \nabla)\right|_{X-\{p\}}$ such that

1) the characteristic polynomial of $\Gamma^{\prime}=\operatorname{res}_{p}\left(\nabla^{\prime}\right)$ has no multiple zeros,

2) if $\lambda, \mu$ are 2 eigenvalues of $\Gamma^{\prime}$, with $\lambda-\mu \in \mathbb{Z}$, then $|\lambda-\mu| \geq M$,

3) $\left(E^{\prime}, \nabla^{\prime}\right)$ is obtained from $(E, \nabla)$ by at most $\frac{N^{3} M}{2}$ elementary $G$-transformations at $p$.

Proof. One orders the roots of the characteristic polynomial of $\Gamma$ in subsets $I_{1}, \ldots, I_{\ell}$

$$
I_{j}=\left\{\lambda_{j, 1}, \ldots, \lambda_{j, m j}\right\}, \text { where } \sum_{j=1}^{\ell} m_{j}=N
$$

such that $0 \leq \lambda_{j, i+1}-\lambda_{j, i} \in \mathbb{N}$, and $\lambda_{j, s}-\lambda_{j^{\prime}, s^{\prime}} \notin \mathbb{Z}$ for $j^{\prime} \neq j$. By taking an eigenvector $e_{1} \in E \otimes k(p)$ for $\lambda_{11}$ and replacing $E$ by $E_{e_{1}}$, one transforms $I_{1}$ to

$$
I_{1}=\left\{\lambda_{1,1}-1, \lambda_{1,2}, \ldots, \lambda_{1, m_{1}}\right\} .
$$

Repeating this $m_{1} M$ times, one replaces $I_{1}$ by

$$
I_{1}=\left\{\lambda_{1,1}-m_{1} M, \lambda_{1,2}, \ldots, \lambda_{1, m_{1}}\right\} .
$$

Since $\lambda_{1,1}-m_{1} M \neq \lambda_{1,2}$, there exists an eigenvector $e_{2}$ with eigenvalue $\lambda_{1,2}$, and repeating the same transformation $\left(m_{1}-1\right) M$ times with $e_{2}$ instead of $e_{1}$ one transforms $\lambda_{1,2}$ to $\lambda_{1,2}-\left(m_{1}-1\right) M$, without changing the other roots of the characteristic polynomial. After $\frac{m_{1}\left(m_{1}-1\right)}{2} M$ steps, one has

$$
I_{1}=\left\{\lambda_{1,1}-m_{1} M, \lambda_{1,2}-\left(m_{1}-1\right) M, \ldots, \lambda_{1, m_{1}-1}-M, \lambda_{1, m_{1}}\right\} .
$$

Repeating this for $I_{2}, \ldots, I_{\ell}$, one needs at most

$$
\left(\sum_{j=1}^{\ell} \frac{m_{j}\left(m_{j}-1\right)}{2}\right) M \leq \frac{N^{3}}{2} \cdot M
$$

steps to satisfy the first and second condition in 1.2.

\section{The Proof of TheOrem 0.2}

Let $E_{0}=0 \varsubsetneqq E_{1} \subset E_{2} \subset \ldots \subset E_{m}=E$ be the Harder-Narasimhan filtration [3] of a rank $N$ vector bundle $E$, uniquely determined by the two conditions:

$$
\mu_{i}=\mu\left(E_{i} / E_{i-1}\right)<\mu_{i-1}
$$

and $E_{i} / E_{i-1}$ semistable, where $\mu(F)=\operatorname{deg}(F) / \operatorname{rank}(F)$ for any vector bundle. 
In order to prove theorem 0.2 we are allowed to replace $E$ by $E(\ell p)$ for $\ell \in \mathbb{Z}$. In fact, $\nabla$ stabilizes $E(\ell p)$ and the residue $\Gamma$ of $\nabla$ in $p$ is replaced by $\Gamma-\ell$ Id. In particular this does not change the difference between two eigenvalues of $\Gamma$. Thus, replacing $E$ by $E(\ell p)$, we may assume that $-1<\mu\left(E_{1}\right) \leq 0$ and consequently that $\operatorname{deg}(E) \leq 0$.

Lemma 2.1. If $\nabla: E \rightarrow \Omega_{X}^{1}(\log \Sigma) \otimes E$ does not stabilize any subbundle, and $-1<\mu\left(E_{1}\right) \leq 0$, then

$$
-N-N^{2}(2 g-2+\sigma) \leq \operatorname{deg}(E) \leq 0
$$

where $g=$ genus of $X$, and $\sigma=|\Sigma|$.

Proof. Let $i_{0}$ to be the minimal $i$ such that the map

$$
\eta_{0}: E_{i} \longrightarrow \Omega_{X}^{1}(\log \Sigma) \otimes E / E_{m-1}
$$

is not 0 . Since $\nabla$ does not stabilize any subbundle, $i_{0} \leq m-1$, thus $\eta_{0}$ is linear and factors through $E_{i_{0}} / E_{i_{0}-1}$. This shows that $\mu_{i_{0}} \leq \mu_{m}+(2 g-2+\sigma)$. By assumption $\nabla$ does not stabilize $E_{i_{0}-1}$. Hence there exists some minimal number $i_{1} \leq i_{0}-1$ such that

$$
\eta_{1}: E_{i} \longrightarrow \Omega_{X}^{1}(\log \Sigma) \otimes E / E_{i_{0}-1}
$$

is not trivial. Then $\eta_{1}$ factors through a linear map

$$
E_{i_{1}} / E_{i_{1}-1} \longrightarrow \Omega_{X}^{1}(\log \Sigma) \otimes E_{j_{1}} / E_{j_{1}-1}
$$

for some $j_{1}$ with $i_{0} \leq j_{1} \leq m$. Consequently

$$
\mu_{i_{1}} \leq \mu_{j_{1}}+(2 g-2+\sigma) \leq \mu_{i_{0}}+(2 g-2+\sigma) \leq \mu_{m}+2(2 g-2+\sigma) .
$$

One obtains inductively

$$
-1 \leq \mu_{1} \leq \mu_{m}+m(2 g-2+\sigma)
$$

and, since $\mu(E) \geq \mu_{m}$ and $N \geq m$, the inequality of lemma 2.1.

Finally, one proves theorem 0.2 in the following more precise form:

Theorem 2.2. Let $(X, E, \nabla, \Sigma)$ be as in theorem 0.2. Assume that

$$
-1<\mu\left(E_{1}\right) \leq 0,
$$

that the characteristic polynomial of $\Gamma=\operatorname{res}_{p}(\nabla)$ has no multiple zeros, and that

$$
|\lambda-\mu| \geq M=N+N^{2}(2 g-2+\sigma)
$$

for different eigenvalues $\lambda$ and $\mu$ of $\Gamma$ with $\lambda-\mu \in \mathbb{Z}$.

Then there is a semistable vector bundle $E^{\prime}$ of degree 0 , and an extension $\nabla^{\prime}$ of $\nabla$ to $E^{\prime}$, such that $\left(E^{\prime}, \nabla^{\prime}\right)$ is obtained from $(E, \nabla)$ by at most $M$ elementary $G$-transformations at $p$.

Proof. We argue by induction on $-\operatorname{deg}(E)$ which is smaller than or equal to $M$ by lemma 2.1.

If $\operatorname{deg}(E)=0, \mu\left(E_{1}\right)=\mu(E)=0$ as $\mu\left(E_{1}\right) \geq \mu(E)$. Thus $E_{1}=E$ and $E$ is semistable of degree 0 . 
Assume now that $\operatorname{deg}(E)<0$. If $\mu\left(E_{1}\right)<0$ as well, then for any elementary $G$ transformation at $p$, and any subsheaf $M \subset E_{w}$, one has

$$
\operatorname{deg}(M) \leq \operatorname{deg}(M \cap E)+1 \leq 0
$$

thus

$$
\operatorname{deg}\left(E_{w}\right)=\operatorname{deg}(E)+1 \text { and }-1 \leq \mu\left(\left(E_{w}\right)_{1}\right) \leq 0 .
$$

Otherwise, $\mu\left(E_{1}\right)=\operatorname{deg}\left(E_{1}\right)=0$. We set $F=E_{1}$ for notational simplicity and denote by $Q$ the quotient $Q=E / F$. We consider an elementary $G$ transformation at $p$ such that the eigenvector $w \in E \otimes k(p)$ maps non-trivially to $Q \otimes k(p)$. One obtains an exact sequence

$$
0 \longrightarrow F \longrightarrow E_{w} \longrightarrow Q_{w} \longrightarrow 0 .
$$

Let $\left(E_{w}\right)_{1}$ be the first bundle in the Harder-Narasimhan filtration of $E_{w}$. One certainly has

$$
-1 \leq \mu\left(E_{1}\right) \leq \mu\left(\left(E_{w}\right)_{1}\right) .
$$

The inequality $\mu\left(\left(E_{w}\right)_{1}\right) \leq 0$ is equivalent to the property that $\operatorname{deg}(M) \leq 0$ for all subsheaves $M \subset E_{w}$. Consider $M \subset E_{w}$ and $M \subset M^{\prime} \subset E_{w}$, where $M^{\prime}$ is the inverse image of $M / F \cap M$ under the projection $E_{w} \rightarrow E_{w} / F \cap M$. As $F \cap M \subset F$, one has $\operatorname{deg}(F \cap M) \leq 0$. Thus

$$
\operatorname{deg}(M) \leq \operatorname{deg}(M / F \cap M)=\operatorname{deg}\left(M^{\prime}\right)+\operatorname{deg}(F)=\operatorname{deg}\left(M^{\prime}\right) .
$$

By definition of $E_{1}=F$, one has $\mu\left((E / F)_{1}\right)<0$ and

$$
\operatorname{deg}((M / F \cap M) \cap Q) \leq-1 .
$$

This shows that

$$
\operatorname{deg}(M) \leq \operatorname{deg}(M / F \cap M) \leq \operatorname{deg}((M / F \cap M) \cap Q)+1 \leq-1+1 \leq 0 .
$$

Thus again

$$
\operatorname{deg}\left(E_{w}\right)=\operatorname{deg}(E)+1 \text { and }-1<\mu\left(\left(E_{w}\right)_{1}\right) \leq 0 .
$$

By induction we obtain the theorem.

\section{Existence of CONNECTIONS}

In this section we lift the unitary connections of the graded pieces of the canonical filtration.

Lemma 3.1 (Compare with [6], lemma 3.5). Let $X$ be an algebraic variety over a field $k$,

$$
0 \longrightarrow S \stackrel{\iota}{\longrightarrow} E \stackrel{p}{\longrightarrow} Q \longrightarrow 0
$$

be an extension of vector bundles given by $u \in H^{1}(X, \mathcal{H o m}(Q, S))$. Let $d_{S}$ and $d_{Q}$ be connections on $S$ and $Q$, respectively.

Then there exists a connection $\nabla$ on $E$ lifting $d_{S}$ and $d_{Q}$ if and only if $0=d u \in H^{1}\left(X, \Omega_{X}^{1} \otimes \mathcal{H o m}(Q, S)\right)$, where $d=\mathcal{H}$ om $\left(d_{Q}, d_{S}\right)$. Two such connections differ by an element in $H^{0}\left(X, \Omega_{X}^{1} \otimes \mathcal{H o m}(Q, S)\right)$.

In particular, if $X$ is projective smooth, $k=\mathbb{C}$, and if $d$ is unitary, then $\nabla$ exists. 
Proof. Let $X=\bigcup U_{i}$ be an affine covering of $X$,

$$
\sigma_{i}:\left.\left.Q\right|_{U_{i}} \longrightarrow E\right|_{U_{i}}, \tau_{i}=\mathrm{Id}-\sigma_{i}:\left.\left.E\right|_{U_{i}} \longrightarrow S\right|_{U_{i}}
$$

be some splitting of $u$ on $U_{i}$. Then

$$
\begin{gathered}
\tau_{j}=\tau_{i}+u_{i j} \circ \pi \\
\sigma_{j}=\sigma_{i}-\iota \circ u_{i j}
\end{gathered}
$$

on $U_{i j}$. Define $\nabla_{i}=d_{S} \circ \tau_{i}+\sigma_{i} \circ d_{Q}$. Then

$$
\nabla_{j}-\nabla_{i} \in H^{0}\left(U_{i j}, \Omega_{X}^{1} \otimes \mathcal{H o m}(Q, S)\right)
$$

is a cocycle. Another choice of $\sigma_{i}$ verifies

$$
\begin{aligned}
\sigma_{i}^{\prime} & =\sigma_{i}-\iota \circ u_{i} \\
\tau_{i}^{\prime} & =\tau_{i}+u_{i} \circ \pi
\end{aligned}
$$

for some $u_{i} \in H^{0}\left(U_{i}, \mathcal{H o m}(Q, S)\right)$. Thus

$$
\begin{aligned}
& \nabla_{i}^{\prime}-\nabla_{i}-d_{S}\left(u_{i} \circ \pi\right)-\iota \circ u_{i} \circ d_{Q} \\
= & d\left(u_{i}\right) \in H^{0}\left(U_{i}, \Omega_{X}^{1} \otimes \mathcal{H o m}(Q, S)\right),
\end{aligned}
$$

and therefore the class $\alpha_{i j}$ of

$$
\nabla_{j}-\nabla_{i} \in H^{1}\left(X, \Omega_{X}^{1} \otimes \mathcal{H o m}(Q, S)\right)
$$

is well defined. If this class vanishes, then in a refinement of $\left(U_{i}\right)$ there are forms $A_{i} \in H^{0}\left(U_{i}, \Omega_{X}^{1} \otimes \mathcal{H o m}(Q, S)\right)$ such that $\nabla_{j}-\nabla_{i}=A_{i}-A_{j}$, thus $\nabla=\nabla_{i}+A_{i}$ is globally defined and $\alpha_{i j}$ is the exact obstruction to the existence of $\nabla$.

On the other hand, the computation in 3.2, with $u_{i}$ replaced by $u_{i j}$, shows at the same time that $\alpha_{i j}=d u_{i j}$.

Let $X$ be a projective curve over $\mathbb{C}$ and $E$ be a semistable bundle of degree 0 on $X$. Then there is a unique filtration, which we call the canonical filtration of $E$, verifying

$$
\begin{gathered}
0=E_{0} \subset E_{1} \subset \ldots \subset E_{m}=E \\
g r_{i} E=E_{i} / E_{i-1}=\text { socle of } E / E_{i-2} .
\end{gathered}
$$

Recall that the socle of $E$ is the maximal semistable subbundle of $E$ which splits as a sum $\bigoplus_{\nu} V_{\nu}$ of stable ones.

$$
\begin{gathered}
\operatorname{Hom}\left(g r_{i} E, E / E_{i}\right)=\operatorname{Hom}\left(g r_{i} E, g r_{i+1} E\right) \\
=\bigoplus \delta_{\nu \mu} \operatorname{Id}_{V_{\nu}}
\end{gathered}
$$

with $g r_{i} E=\bigoplus_{\nu} V_{\nu}, g r_{i+1} E=\bigoplus_{\mu} V_{\mu}$ for stable bundles $V_{\nu}$ and $V_{\mu}$.

On the other hand, over $\mathbb{C}$, there is a unique unitary connection $d_{i}$ on $g r_{i} E$ by the Narasimhan-Seshadri correspondence [5].

Proposition 3.2. Let $E$ be a semistable bundle of degree 0 on a complex projective curve, and $E_{i}$ be its canonical filtration. Then there is a connection $\nabla$ on $E$ respecting the canonical filtration and lifting the unitary connections $d_{i}$ on $E_{i} / E_{i-1}$. 
Proof. Since $\mathcal{H o m}\left(d_{m}, d_{m-1}\right)$ is unitary, there is a connection $d_{E / E_{m-1}}$ lifting $d_{m}$ and $d_{m-1}$ by lemma 3.1. Assume inductively that $d_{E / E_{\ell}}$ exists. We want to see that

$$
d: H^{1}\left(X, \mathcal{H o m}\left(E / E_{\ell}, g r_{\ell} E\right)\right) \longrightarrow H^{1}\left(X, \Omega_{X}^{1} \otimes \mathcal{H o m}\left(E / E_{\ell}, g r_{\ell} E\right)\right)
$$

kills the extension of $E / E_{\ell}$ by $g r_{\ell} E$ given by the canonical filtration, where $d=\mathcal{H o m}\left(d_{E / E_{\ell}}, d_{\ell}\right)$. We show directly that $d$ itself vanishes. Its dual is the differential

$$
d^{*}: H^{0}\left(X, \mathcal{H o m}\left(g r_{\ell} E, E / E_{\ell}\right)\right) \longrightarrow H^{0}\left(X, \Omega_{X}^{1} \otimes \mathcal{H o m}\left(g r_{\ell}, E / E_{\ell}\right)\right) .
$$

By the equation 3.4, and the fact that $d^{*} \operatorname{lifts} \mathcal{H o m}\left(d_{\ell}, d_{\ell+1}\right)$, one has $d^{*}=$ $\operatorname{Hom}\left(d_{\ell}, d_{\ell+1}\right)=0$.

Lemma 3.3. Let $X$ be a smooth projective variety defined over a field $k$ of characteristic zero, $D$ be a smooth irreducible divisor, $L$ be an invertible sheaf $L$, and let $\nabla: L \rightarrow \Omega_{X}^{1}(\log D) \otimes L$ be a connection. Then the residue $\operatorname{res}_{D}(\nabla)$ is $m$. id for a rational number $m$. Moreover, if $X$ is a curve, $m$ is an integer.

Proof. Since $X$ is projective, we may write $L=\mathcal{O}\left(A_{1}-A_{2}\right)$ where $A_{i}$ are smooth divisors meeting transversally. Thus $L$ carries the trivial connection $d_{A}$ with $\operatorname{res}_{A_{i}}\left(d_{A}\right)=(-1)^{i} \cdot \operatorname{Id}_{\left.L\right|_{A_{I}}}$. Hence $\omega:=\nabla-d_{A} \in H^{0}\left(X, \Omega_{X}^{1}\left(\log \left(A_{1}+\right.\right.\right.$ $\left.\left.A_{2}+D\right)\right)$ with

$$
m:=\operatorname{res}_{D}(\omega)=\operatorname{res}_{D} \nabla, \quad \operatorname{res}_{A_{i}}(\omega)=-\operatorname{res}_{A_{i}}\left(d_{A}\right) .
$$

Let $C$ be an ample smooth curve, meeting $D, A_{1}$ and $A_{2}$ transversally. Then

$$
-\left(C . A_{1}\right)+\left(C . A_{2}\right)+m \cdot(C . D)=\sum_{q \in C \cap\left(A_{1} \cup A_{2} \cup D\right)} \operatorname{res}_{q}(\omega)=0
$$

and consequently $m \in \mathbb{Q}($ or $m \in \mathbb{Z}$, if $\operatorname{dim}(X)=1)$.

Lemma 3.4. Let $X$ be a smooth projective variety over a field $k$ of characteristic zero, let $D=\sum_{i=1}^{\rho} D_{i}$ be a normal crossing divisor and

$$
\left.\nabla: V \rightarrow \Omega_{X}^{1} \log D\right) \otimes V
$$

a connection on a locally free sheaf $V$. Assume that the eigenvalues of $\operatorname{res}_{D_{i}}(\nabla)$ are zero for $i=2, \ldots, \rho$ and that the sum of the eigenvalues of $\operatorname{res}_{D_{1}}(\nabla)$ does not lie in $\mathbb{Q}-\{0\}$ (or not in $\mathbb{Z}-\{0\}$, if $X$ is a curve). Then $\bigwedge^{\max } V$ is numerically trivial.

Proof. $\nabla$ induces a connection

$$
\nabla^{\prime}: \bigwedge^{\max } V \longrightarrow \Omega_{X}^{1}(\log D) \otimes \bigwedge^{\max } V .
$$

$\operatorname{res}_{D_{i}}\left(\nabla^{\prime}\right)=0$ for $i=2, \ldots, \rho$, and the image of $\nabla^{\prime}$ lies in $\Omega_{X}^{1}\left(D_{1}\right) \otimes \bigwedge^{\max } V$. By $3.3 \operatorname{res}_{D_{1}}\left(\nabla^{\prime}\right)$ must be a rational number (or an integer), hence 0 , and $\nabla^{\prime}$ induces a connection with values in $\Omega_{X}^{1} \otimes \bigwedge^{\max } V$. 


\section{Existence OF IRREDUCIBLE CONNECTIONS}

Let $E$ be a semistable bundle of rank $N$ on the curve $X$ and let

$$
\nabla: E \rightarrow \Omega_{X}^{1} \otimes E
$$

be a connection. In this section we want to construct a different connection $\nabla^{\prime}: E \rightarrow \Omega_{X}^{1}(\log \Sigma) \otimes E$, where $\Sigma=\sum_{i=1}^{\mu} p_{i}$ is a reduced divisor in $X$, such that $\operatorname{Ker}\left(\left.\nabla^{\prime}\right|_{X-\Sigma}\right)$ is an irreducible local system. If $X$ is defined over $\mathbb{C}$ this construction and 3.2 imply proposition 0.3 .

Proposition 4.1. Assume that $E$ is not isomorphic to the direct sum $L^{\oplus N}$ for some $L \in \operatorname{Pic}^{0}(X)$ and let $p, q \in X$ be two different points. Then there exists $\varphi \in \operatorname{Hom}\left(E, \Omega_{X}^{1}(\log (p+q)) \otimes E\right)$ such that $\operatorname{Ker}\left(\left.\nabla^{\prime}\right|_{X-p-q}\right)$ is irreducible for $\nabla^{\prime}=\nabla+\varphi$.

Proof. By assumption there exists a surjection $\tau: E \rightarrow S$ for some bundle $S$ on $X$ of rank $s \geq 2$ such that one of the following properties holds true:

i) $S$ is stable

ii) $S=L_{1} \oplus L_{2}$ for $L_{1} \nRightarrow L_{2}$ and $L_{i} \in \operatorname{Pic}^{0}(X)$

iii) $0 \rightarrow T \rightarrow S \rightarrow L^{\oplus \ell} \rightarrow 0$ is an extension of $L^{\oplus \ell}$, for $L \in \operatorname{Pic}^{0}(X)$ with a stable bundle $T$, such that the induced map

$$
H^{0}\left(X, \mathcal{O}_{X}^{\oplus \ell}\right) \longrightarrow H^{1}\left(X, T \otimes L^{-1}\right)
$$

is injective.

In fact, let $F_{0}^{*}=\{0\} \subset F_{1}^{*} \subset \ldots \subset F_{m}^{*}=E^{*}$ be the canonical filtration of the dual bundle and

$$
F_{0}=\{0\} \subset F_{1}=\left(E^{*} / F_{m-1}^{*}\right)^{*} \subset \ldots \subset F_{m-1} \subset\left(E^{*} / F_{1}^{*}\right)^{*} \subset F_{m}=E
$$

the dual filtration. If $F_{m} / F_{m-1}$ contains no semistable bundle $S$ as in i) or ii) it is a direct sum $L^{\oplus \ell^{\prime}}$, for some $\ell^{\prime} \geq 1$. In this case,

$$
F_{m-1} / F_{m-2} \longrightarrow E / F_{m-2} \longrightarrow L^{\oplus \ell^{\prime}}
$$

is a non-trivial extension and for each direct factor $T$ of $F_{m-1} / F_{m-2}$ one obtains a surjection from $E$ to a non-trivial extension

$$
0 \longrightarrow T \longrightarrow S^{\prime} \longrightarrow L^{\oplus \ell^{\prime}} \longrightarrow 0 .
$$

Leaving out direct factors of $S^{\prime}$, which are isomorphic to $L$, one obtains $S$ as in iii).

For any bundle $F$ on $X$ write $F_{q}=F \otimes k(q)$. In order to construct a basis of $E_{q}$ we fix a basis of $S_{q}$, case by case:

i) $\bar{v}_{1}, \ldots, \bar{v}_{m-1}, \bar{v}_{N}$ is any basis of $S_{q}$.

ii) $\bar{v}_{1}, \bar{v}_{N}$ is a basis of $S_{q}$ with $\bar{v}_{1} \notin\left(L_{i}\right)_{q}$, for $i=1,2$.

iii) $\bar{v}_{1}, \ldots, \bar{v}_{m-1}, \bar{v}_{N}$ is a basis of $S_{q}$, such that $T_{q} \not \subset<\bar{v}_{1}, \ldots, \bar{v}_{m-1}>$.

Let $K=\operatorname{Ker}(\tau: E \rightarrow S)$ and

$$
0 \longrightarrow K_{q} \longrightarrow E_{q} \stackrel{\tau_{q}}{\longrightarrow} S_{q} \longrightarrow 0
$$

the induced sequence of vector spaces. 
Let $v_{m}, \ldots, v_{N-1}$ be a basis of $K_{q}$, and $v_{j} \in \tau_{q}^{-1}\left(\bar{v}_{j}\right)$, for $j=1, \ldots, m-1, N$. Then $v_{1}, \ldots, v_{N}$ is a basis of $E_{q}$. By Serre duality

$$
h^{1}\left(X, \mathcal{E} n d(E) \otimes \Omega_{X}^{1}(\log p)\right)=h^{0}(X, \mathcal{H o m}(E, E(-p))=0,
$$

hence the residue map

$$
H^{0}\left(X, \mathcal{E} n d(E) \otimes \Omega_{X}^{1}(\log (p+q))\right) \stackrel{\operatorname{res}_{q}}{\longrightarrow} \operatorname{End}\left(E_{q}\right)
$$

is surjective. Choose $\varphi \in \operatorname{End}\left(E, \Omega_{X}^{1}(\log (p+q)) \otimes E\right)$ such that $\operatorname{res}_{q}(\varphi)$ is one Jordan block for the eigenvalue 0 , with respect to $v_{1}, \ldots, v_{N}$. In particular, the only $\operatorname{res}_{q}(\varphi)$ invariant subspaces of $E_{q}$ are of the form $\operatorname{Ker}\left(\operatorname{res}_{q}(\varphi)^{\iota}\right)$.

Let $\lambda_{1}, \ldots, \lambda_{\nu}$ be the eigenvalues of $\operatorname{res}_{p}(\varphi)$. Replacing $\varphi$ by $\pi \cdot \varphi$ for some $\pi \notin \mathbb{Q}\left(\lambda_{1}, \ldots, \lambda_{\nu}\right)$ we may assume that no linear combination $\Sigma \rho_{i} \lambda_{i} \in \mathbb{Q}-\{0\}$ for $\rho_{i} \in \mathbb{Q}$.

Let $V \subset E$ be a subbundle such that $\nabla^{\prime}(V) \subset \Omega_{X}^{1}(\log (p+q)) \otimes V$, for $\nabla^{\prime}=\nabla+\varphi$. By $3.4 \operatorname{deg}(\mathrm{V})=0$, hence $V$ is a semistable subbundle of $E$, and the image $B$ of $V$ in $S$ is zero or a semistable subbundle of $S$.

Since $\operatorname{res}_{q}\left(\nabla^{\prime}\right)=\operatorname{res}_{q}(\varphi)$, for some $\iota \geq 1$

$$
V_{q}=\operatorname{Ker}\left(\operatorname{res}_{q}(\varphi)^{\iota}\right)=<v_{1}, \ldots, v_{\iota}>\subset E_{q} .
$$

In particular $B \neq 0$. Obviously $B=S$ in case i). In case ii) we remark that $v_{1} \in B_{q}$ and obtain $B=S$, as well.

If in case iii) $B \neq S$, then $B_{q}=<v_{1}, \ldots, v_{\iota}>$ for $\iota \leq m-1$ and $B \cap T \neq T$. Since the degree of $B$ is zero, and since $B /(B \cap T) \subset L^{\oplus \ell}, B \cap T=0$. Then $B \simeq L^{\oplus \iota}$ and the composite

$$
H^{0}\left(X, B \otimes L^{-1}\right) \hookrightarrow H^{0}\left(X, \mathcal{O}_{X}^{\oplus \ell}\right) \longrightarrow H^{1}\left(X, T \otimes L^{-1}\right)
$$

zero, contradicting the assumptions made.

Hence $B=S$ in all cases, and $v_{n} \in V_{q}$. Therefore $V_{q}=E_{q}$ and $V=E$.

If $E=L^{\oplus N}$, then in order to find some $\varphi$, with $\operatorname{Ker}\left(\nabla+\left.\varphi\right|_{X-\Sigma}\right)$ irreducible, one needs three points $p, q_{1}, q_{2}$. In fact, choosing the "canonical" basis $v_{1}^{(i)}, \ldots v_{N}^{(i)}$ in $E_{q_{i}}$, induced by the direct sum decomposition, one has again a surjection

$$
\operatorname{End}\left(E, \Omega_{X}^{1}\left(\log \left(p+q_{1}+q_{2}\right)\right)\right) \longrightarrow M(N \times N, \mathbb{C}) \oplus M(N \times N, \mathbb{C}) .
$$

Let us choose two nilpotent matrices $M_{1}$ and $M_{2}$ with $M_{i}^{N-1} \neq 0$ in such a way, that the (unique) eigenvector of $M_{1}$ does not lie in $\operatorname{Ker}\left(M_{2}^{N-1}\right)$. Repeating the argument used in the proof of 4.1 one obtains:

Proposition 4.2. Let $\Sigma=q_{1}+q_{2}+p$ be a reduced divisor and $E$ be a semistable bundle with connection $\nabla$. Then for some $\varphi \in \operatorname{Hom}\left(E, \Omega_{X}^{1}(\log \Sigma) \otimes E\right)$ the local system $\operatorname{Ker}\left(\left.(\nabla+\varphi)\right|_{X-\Sigma}\right)$ is irreducible.

Under stronger condition on the structure of $E$, it is possible to choose $\Sigma=p$, as we illustrate in two examples on an elliptic curve $X$.

Example 4.3. Let $L \in \operatorname{Pic}^{0}(X), L \neq \mathcal{O}, E=L \oplus \mathcal{O}$. Take $\Sigma=\{p\}$ a point. Then choose

$$
\nabla=d+\left(\begin{array}{ll}
\alpha & \beta \\
\gamma & \delta
\end{array}\right)
$$


where $d$ is the sum of the unitary connections on $L$ and $\mathcal{O}$,

$$
\begin{gathered}
\alpha, \delta \in H^{0}\left(X, \Omega_{X}^{1}(\log \Sigma)\right)=H^{0}\left(X, \Omega_{X}^{1}\right) \\
\gamma \in H^{0}\left(X, L^{-1} \otimes \Omega_{X}^{1}(\log \Sigma)\right)-H^{0}\left(X, L^{-1} \otimes \Omega_{X}^{1}\right) \\
\beta \in H^{0}\left(X, L \otimes \Omega_{X}^{1}(\log \Sigma)\right)-H^{0}\left(X, L^{-1} \otimes \Omega_{X}^{1}\right) .
\end{gathered}
$$

Assume $\operatorname{res}_{q} \gamma=\lambda, \operatorname{res}_{q} \beta=\mu$ are chosen such that $x^{2}-\lambda \cdot \mu$ has no zero in $\mathbb{Q}$. If $V \subset E$ of rank 1 is stabilized by $\nabla$, then $\operatorname{residue}_{p}\left(\left.\nabla\right|_{V}\right) \notin \mathbb{Q}$. This contradicts lemma 3.3 .

Example 4.4. Let $X$ be an elliptic curve and

$$
0 \longrightarrow \mathcal{O}_{S} \stackrel{\iota}{\longrightarrow} E \stackrel{\pi}{\longrightarrow} \mathcal{O}_{Q} \longrightarrow 0
$$

be the non-trivial extension of $\mathcal{O}_{X}$ by $\mathcal{O}_{X}$. As we have seen in 3.2, there exists a connection $\nabla$ on $E$, lifting $d: \mathcal{O}_{X} \rightarrow \Omega_{X}^{1}$. As

$$
h^{0}(X, \mathcal{E} n d(E))=h^{1}(X, \mathcal{E} n d(E))=2 \text { and } h^{1}(X, \mathcal{E} n d(E(p)))=0
$$

for any point $p$, whereas $H^{0}\left(X, \mathcal{O}_{X}\right)=H^{0}(X, \mathcal{O}(p))$, the image of

$$
\operatorname{res}_{p}: \operatorname{Hom}(E, E(p))=\operatorname{Hom}\left(E, E \otimes \Omega_{X}^{1}(\log p)\right) \longrightarrow M(2 \times 2, k)
$$

is a two-dimensional space of matrices of trace 0 . In particular the image contains some lower triangular matrix

$$
M=\left(\begin{array}{cc}
\alpha & 0 \\
\gamma & -\alpha
\end{array}\right) \neq 0
$$

with respect to a basis $v_{1}, v_{2}$ with $v_{1} \in \iota(k(p))$. Choose $\phi \in \operatorname{Hom}(E, E(p))$ and $\lambda \in k$ with $\operatorname{res}_{p} \phi=\lambda \cdot M$, such that $\lambda \alpha \notin \mathbb{Z}-\{0\}$. By 3.4 a rank 1 subbundle $V \subset E$ with $\nabla(V) \subset \Omega_{X}^{1}(\log \Sigma) \otimes V$ is numerically trivial, hence equal to $\iota\left(O_{X}\right)$. Then $\alpha$ and $\gamma$ are both zero, contradicting the assumption $M \neq 0$.

\section{REFERENCES}

[1] Anosov, D. A.; Bolibruch, A.A.: The Riemann-Hilbert Problem, Aspects of Mathematics 22 (1994), Vieweg Verlag.

[2] Gabber, O: letter to A. Beauville, March 1993.

[3] Harder, G.; Narasimhan, M. S.: On the cohomology groups of moduli spaces, Math. Ann. 212 (1975) 215 - 248.

[4] Lekaus, S.: Diplomarbeit, Universität Essen 1998.

[5] Narasimhan, M. S.; Seshadri, C. S.: Stable and unitary bundles on a compact Riemann surface, Ann. Math. 82 (1965), 540 - 567.

[6] Simpson, C.: Higgs bundles and local systems, Publ. Math. IHES 75 (1992), 5-95.

Universität Essen, FB6 Mathematik, 45117 Essen, Germany

E-mail address: esnault@uni-essen.de

E-mail address: viehweg@uni-essen.de 